\section{Presença de trialometanos \\ na água e efeitos adversos na \\ gravidez}

\section{Trihalomethanes in drinking water and adverse effects on pregnancy}

\section{Sônia Maria dos Santos'}

\section{Nelson Gouveia"}

' Faculdade de Medicina da Universidade de São Paulo - Universidade Regional de Blumenau.

" Departamento de Medicina Preventiva da Faculdade de Medicina da Universidade de São Paulo.

Correspondência: Sônia Maria dos Santos. R. Dr. Luiz de Freitas Melro, 6, apto. 602 - Blumenau SC. E-mail: soniamds@terra.com.br

\section{Resumo}

Este trabalho constituiu um estudo do tipo transversal, que objetivou avaliar a possível associação entre a exposição a trialometanos presentes na rede pública de abastecimento de água da região metropolitana de São Paulo e a ocorrência de desfechos adversos na gravidez. Para a realização deste estudo, foram selecionados 19 municípios da região metropolitana de São Paulo que eram abastecidos por apenas uma estação de tratamento de água, no período de 1998 a 2002. Partiu-se da verificação dos níveis de trialometanos na água de abastecimento e da análise da prevalência de baixo peso ao nascer, prematuridade, anomalias congênitas, defeitos no tubo neural e no sistema nervoso central, nos recém-nascidos dos municípios estudados, para se analisar a associação entre a exposição a trialometanos e a ocorrência de desfechos adversos na gravidez. A população estudada consistiu em todas as mulheres grávidas e seus filhos recém-nascidos, residentes nos municípios selecionados durante o período de estudo, que tiveram suas declarações de nascido vivo registradas no Sistema de Informações sobre Nascidos Vivos (SINASC). Os níveis de trialometanos foram tratados como categorias. A análise descritiva foi seguida pela análise univariada, e esta pela análise multivariada. Para expressar as possíveis associações dos desfechos pesquisados com os trialometanos foi utilizado o teste qui-quadrado, seguido da estimativa das razões de chance (odds ratio - OR) com intervalos de $95 \%$ de confiança. O controle das variáveis de confusão se deu através da análise de regressão logística múltipla não condicional, seguindo os procedimentos de Hosmer e Lemeshow (2000). As variáveis que apresentaram nível de significância estatística $(p \leq 0,2)$ na análise univariada foram inseridas no modelo múltiplo. Os resultados obtidos apontaram para uma associação entre malformação e prematuridade relacionadas a trialometanos, o que suscita análises mais profundas dadas as implicações de tal fato sobre a Saúde Pública e levanta uma relevante discussão sobre os efeitos benéficos da cloração em comparação com o risco reduzido de desfechos adversos para a gravidez, indicando, entretanto, que seria recomendável um melhor monitoramento dos níveis de trialometanos.

Palavras-chave: Trialometanos. Prematuridade. Baixo peso ao nascer. Anomalias congênitas. Defeitos no tubo neural. Defeitos no sistema nervoso central. 


\section{Abstract}

This paper describes a cross-sectional study that aimed to evaluate the possible association between exposure to trihalomethanes present in public water supplies in the metropolitan region of São Paulo and the occurrence of adverse outcomes in pregnancy. In order to perform the research, 19 municipalities located in the metropolitan area of São Paulo that were supplied by only one water treatment plant, from 1998 to 2002, were selected. The starting point was the verification of trihalomethane levels in public water supplies and the analysis of the prevalence of low birth weight, preterm delivery, congenital anomalies, neural tube and nervous system defects in newborns of the municipalities that were involved in the study, in order to analyze the association between exposure to trihalomethanes and the occurrence of adverse outcomes in pregnancy. The population that was studied consisted of all pregnant women and their newborn children who were living in the selected municipalities during the research period, and had their birth certificates registered in the Information System on Live Births (SINASC). The levels of trihalomethanes were treated as categories. The descriptive analysis was followed by a univariate analysis and then by a multivariate analysis. To express the possible associations of the research outcomes with trihalomethanes, the chi-square test was used followed by the estimation of odds-ratio with a $95 \%$ confidence interval. Confounding variables were controlled through the analysis of unconditional multiple logistic regression, following the procedures of Hosmer and Lemeshow (2000). The variables that showed statistical significance $(p \leq 0.2)$ in univariate analysis were entered into the multivariate mode. The results pointed out an association between possible risk of prematurity and malformation related to trihalomethanes, raising the need for in-depth studies, due to the implications of that fact in public health. The results raise a discussion on the relevant benefits of chlorination in comparison to the reduced risk of adverse outcomes in pregnancy; they also suggest that better monitoring of trihalomethane levels in public water supplies would be advisable.

Keywords: Trihalomethanes. Low birthweight. Preterm delivery. Congenital anomalies. Neural tube defects. Nervous system defects.

\section{Introdução}

A saúde pública no Brasil sempre teve a sua atenção voltada para os problemas de saúde relacionados a exposições ambientais. Todavia, seu interesse principal estava inicialmente concentrado na contaminação da água por agentes biológicos. A implementação, ainda que parcial, de medidas sanitárias tradicionais ocasionou um declínio da morbimortalidade causada pelos patógenos biológicos veiculados pela água, o que provocou o aumento da importância das doenças causadas por agentes físicos e químicos.

Estima-se que, no mundo todo, cerca de quatro bilhões de metros cúbicos de contaminantes, provenientes principalmente de efluentes industriais, compostos químicos de uso agrícola, efluentes domésticos e outros, atinjam o solo a cada ano e, consequentemente, a água. Esses contaminantes, uma vez no ambiente, podem sofrer transformações físicas, químicas e combinação com outros compostos químicos, o que facultativamente intensifica ou diminui sua toxicidade a seres humanos e organismos vivos $^{1}$.

No Brasil, o controle da exposição aos fatores de risco biológicos tem se resumido ao tratamento da água, processo que consta das seguintes fases: decantação, filtração e cloração. A cloração representa um beneficio indiscutível à saúde humana, uma vez que o cloro, em qualquer de seus compostos, é capaz de destruir e tornar inativos os organismos causadores de enfermidades. Sua aplicação é simples, exigindo equipamentos de baixo custo; a determinação de sua concentração é fácil e é relativamente seguro ao homem nas dosagens habitualmente adotadas $(2,0 \mathrm{mg} / \mathrm{L})$ para a desinfecção da água ${ }^{2,3}$. Entretanto, o cloro utilizado para desinfecção da água pode se associar à matéria orgânica geralmente presente nas águas superficiais captadas, gerando subprodutos, dentre eles os trialometanos.

No Brasil, Fernicola e Azevedo ${ }^{4}$ encontraram trialometanos em amostras de 
água bruta, de água tratada nas estações de tratamento, nos reservatórios e nos pontos de distribuição de alguns sistemas de abastecimento do Estado de São Paulo, entre agosto e outubro de 1980. Nessa análise, observaram um aumento nas concentrações de trialometanos detectados, em função do tempo que a água ficava armazenada. Quanto maior o tempo de contato da matéria orgânica com o cloro, maior a produção de trialometanos ${ }^{5}$.

Vários estudos ${ }^{6-10}$ têm demonstrado uma associação entre a presença de trialometanos na água de abastecimento, em concentrações que variam entre $80 \mathrm{e}$ $100 \mu \mathrm{g} / \mathrm{L}$, e efeitos na saúde da população. Também tem-se focalizado a relação entre a concentração de trialometanos e efeitos adversos no aparelho reprodutor, bem como no desenvolvimento fetal.

Hwang e Jaakkola ${ }^{10}$, em Maryland, apontaram a existência de evidências consistentes do efeito da exposição aos trialometanos sobre defeitos no tubo neural e no sistema urinário. Em Santa Clara, Califórnia, ao se analisar mulheres grávidas expostas à água de torneira, encontrou-se excesso de aborto espontâneo entre mulheres que usavam qualquer tipo de água de torneira durante o terceiro mês de gestação, quando comparadas com as que reportaram nenhum consumo de água de torneira ${ }^{6}$. Em estudos realizados em Iowa ${ }^{11} \mathrm{e}$ em New Jersey ${ }^{12}$, obteve-se, respectivamente, risco moderadamente mais alto de retardo no crescimento intrauterino associado a concentrações de clorofórmio maiores do que $9 \mu \mathrm{g} / \mathrm{L}$ (na água fornecida pela rede de abastecimento) e associações positivas para pequeno para idade gestacional, para baixo peso ao nascer e nascimentos a termo, e para defeitos de nascimentos em geral para níveis de trialometano $>100 \mu \mathrm{g} / \mathrm{L}$. Igualmente Waller, Swan, Delorenze e Hopkins ${ }^{13}$ encontraram aumento dos números de casos de perda fetal entre 12 e 28 semanas associado à exposição a trialometanos. Outros estudos realizados nos Estados Uni$\operatorname{dos}^{14-17}$ também associaram a exposição a trialometanos a risco de aborto, defeito no tubo neural e baixo peso ao nascer. Além disso, em um estudo de caso-controle realizado em Ontário, Canadá1 ${ }^{18}$, ao se examinar a associação de trialometanos e risco de natimorto, concluiu-se que as mulheres expostas a $80 \mu \mathrm{g} / \mathrm{L}$ de trialometanos, ou mais, apresentavam um risco duas vezes maior de terem natimortos do que as não expostas.

Enquanto a associação entre trialometanos e problemas de saúde em humanos tem sido exaustivamente estudada em outros países ${ }^{19}$, no Brasil, os poucos estudos realizados ${ }^{1,3}$ limitam-se aos aspetos relacionados à qualidade da água. Portanto, percebendo-se esta lacuna em relação a uma análise mais aprofundada do efeito desses compostos sobre a saúde humana, o presente trabalho objetivou verificar a presença de trialometanos na água de abastecimento fornecida aos municípios da região metropolitana de São Paulo e a sua associação com aspectos relacionados aos recém-nascidos: baixo peso ao nascer, prematuridade, malformação congênita, defeito no tubo neural e defeito no sistema nervoso central.

\section{Método}

Esta pesquisa constituiu um estudo do tipo transversal, para o qual foram selecionados 19 municípios da região metropolitana de São Paulo que são abastecidos por apenas uma Estação de tratamento de água - ETA (Quadro 1).

A população estudada consistiu em todas as mulheres grávidas e seus filhos recém-nascidos, residentes nos municípios selecionados durante o período de estudo (1998 a 2002), que tiveram suas Declarações de Nascido Vivo (DN) registradas no SINASC (Sistema de Informação de Nascidos Vivos). Neste documento, preenchido na maternidade onde a criança nasceu ou recebeu atendimento imediato após o nascimento, há informações quanto às características epidemiológicas relativas aos recém-nascidos, tais como: data de nascimento, sexo, índice de Apgar, peso ao nascer e presença de malformações congênitas e/ou anomalia 
Quadro 1 - Relação dos sistemas de abastecimento e dos municípios por eles abastecidos. Chart 1 - Water supply systems and municipalities served by them.

\begin{tabular}{|c|c|}
\hline SISTEMAS DE ABASTECIMENTO & MUNICíPIOS \\
\hline Guaraú & Caieras \\
& Osasco \\
& Carapicuíba \\
& Franco da Rocha \\
& Francisco Morato \\
& São Caetano do Sul \\
\hline Alto da Boa Vista & Taboão da Serra \\
\hline Alto Tietê & Arujá \\
& Ferraz de Vasconcelos \\
& Itaquaquecetuba \\
& Poá \\
& Diadema \\
\hline Alto Cotia & Embu Guaçú \\
& Itapecerica da Serra \\
& Vargem Grande Paulista \\
\hline Baixa Cotia & Itapevi \\
& Jandira \\
\hline Casa Grande & Ribeirão Pires \\
\hline Rio Grande & São Bernardo do Campo \\
\hline
\end{tabular}

Fonte: SABESP/Source: SABESP

cromossômica; e informações referentes à duração da gestação, tipo de gravidez, tipo de parto, número de consultas de pré-natal e dados relativos à mãe (idade, estado civil, escolaridade, ocupação, paridade e local de residência). No Estado de São Paulo, a segunda via da DN é encaminhada à Fundação SEADE (Sistema Estadual de Análise de Dados), e os dados nela contidos são computados. Estima-se uma cobertura de 95\% do total de nascimentos efetivamente ocorridos $^{20}$.

As informações referentes aos parâmetros de qualidade da água, mensuradas pela Companhia de Saneamento Básico do Estado de São Paulo (SABESP), foram obtidas no Centro de Vigilância Sanitária (CVS) da Secretaria de Estado da Saúde (SES).

As medidas referentes aos trialometanos são realizadas no mínimo três vezes ao ano, através de coletas e análises realizadas de três em três meses. De acordo com a portaria $n^{\circ} 1469 / 2000$, em algumas ETAS essa medidas podem ser tornar mais frequentes, com o objetivo de manter e controlar a qualidade da água produzida e distribuída. Os níveis de trialometanos são fornecidos em $\mu \mathrm{g} / \mathrm{L}$ pela SABESP que, após a medição, envia-os ao Centro de Vigilância Sanitária do Estado de São Paulo.

Normalmente, por ocasião dos períodos de chuva, as concentrações de matéria orgânica presentes nas águas de rios e córregos tendem a aumentar e, por ocasião do beneficiamento da água, esta matéria orgânica entra em contato com o cloro, acarretando a formação dos trialometanos. Esse processo não ocorre de forma regular em todas as ETAS. Tal constatação fez com que se optasse pela utilização dos níveis máximos de trialometanos, por trimestre, de 1998 a 2002, e não da média dos níveis de trialometanos.

As definições adotadas para as variáveis estudadas foram:

- Baixo peso ao nascer - recém-nascido a termo (idade gestacional entre 37-41 semanas de gestação) com peso ao nascer abaixo de $2500 \mathrm{~g}^{21}$;

- Prematuridade - RN cuja duração da 
gestação foi inferior a 37 semanas $^{21}$;

- Malformações congênitas (CID-10 - Q)

- foram consideradas, no âmbito deste trabalho, todas as malformações do Grupo Q, do CID10 e, dentro deste grupo, as malformações do SNC e dentre estas os defeitos no tubo neural.

Neste estudo considerou-se a exposição da mãe ao valor máximo de trialometanos medido na ETA que abastecia o município de sua residência, durante diversos períodos de sua gravidez. Para cada trimestre, caso houvesse somente uma medida, essa era então selecionada. Caso houvesse duas ou mais medidas, a maior entre elas era selecionada. A partir daí foram verificadas as datas de nascimento dos $\mathrm{RN}$ e número de semanas de gestação. Se o RN tivesse nascido a termo voltava-se nove meses atrás em relação ao nascimento e verificava-se o nível máximo de trialometanos na ETA de residência da mãe neste trimestre. Se o RN tivesse nascido pré-termo de oito meses, voltava-se oito meses atrás e verificava-se o nível máximo de trialometanos no trimestre correspondente ao primeiro trimestre de gestação e assim por diante, respeitando sempre o período de gestação e retornando ao primeiro trimestre desta. O mesmo procedimento foi adotado quando se buscou os outros trimestres. Quando o foco era o segundo trimestre, voltava-se, então, seis meses atrás e quando o foco era o último trimestre voltava-se três meses.

$\mathrm{O}$ foco direcionado ao primeiro trimestre da gestação justifica-se pela constatação de que o período de indução de agentes químicos capazes de influenciar malformações neurológicas ocorre entre o momento da fecundação e o $23^{\circ}$ dia de gestação ${ }^{12,22}$. No que diz respeito ao baixo peso, o foco de pesquisa foi direcionado ao $2^{\circ} \mathrm{e} 3^{\circ}$ trimestre do período gestacional ${ }^{23}$, pois é nessa fase que a exposição da mãe à água com trialometanos pode vir a influenciar no ganho de peso. Em relação à prematuridade, o foco foi direcionado à análise do primeiro trimestre de gravidez, uma vez que RN nascidos no segundo e terceiro trimestre de gravidez estavam inclusos na classificação de prétermos, já que no âmbito desta pesquisa são consideradas prematuras as crianças nascidas antes da $37^{a}$ semana de gestação, e, por isso, não se justificava realizar uma coleta de dados correspondente aos dois últimos trimestre de gravidez.

Os dados obtidos foram armazenados no programa Excel 2000, exportados para o programa SPSS 10.0, categorizados, e, em seguida, procedeu-se à análise descritiva. Nesta última, foram observados os níveis de trialometanos nas diferentes ETAs para os anos de 1998 a 2002, para cada um dos 19 municípios previamente selecionados, nos anos de 2000 a 2002. Em seguida, procurouse verificar a ocorrência de baixo peso ao nascer e prematuridade para os anos de 1998 a 2002 e o número de casos de malformação congênita, defeitos no sistema nervoso central e defeitos no tubo neural nos anos de 2000 a 2002 - pois para estas variáveis não havia registros de dados sistematizados antes de 2000 - nos 19 municípios selecionados. Foram realizados cálculos de distribuição de frequência para os eventos acima citados e também foram calculadas taxas/1000 nascidos vivos.

Para expressar as possíveis associações entre os desfechos estudados e a exposição aos trialometanos na gravidez, foi utilizado o teste qui-quadrado, seguido da estimativa das razões de chance (odds ratio-OR) e respectivos intervalos de $95 \%$ de confiança. O controle das variáveis de confusão se deu através da análise de regressão logística múltipla não condicional, seguindo os procedimentos de Hosmer e Lemeshow $^{24}$. As variáveis que apresentaram nível de significância estatística $(\mathrm{p} \leq 0,2)$ na análise univariada foram inseridas no modelo múltiplo. Adotou-se o procedimento passo a passo para a seleção das variáveis, iniciando o modelo pela variável de maior significância estatística e obedecendo a ordem decrescente para inclusão das demais. Foram mantidas as variáveis que apresentaram nível de significância $(\mathrm{p}<0,05)$. Foram realizadas análises múltiplas para os três trimestres de gestação separadamente. 


\section{Resultados}

Os níveis máximos de trialometanos mensurados pela SABESP nas diferentes ETAS que abastecem os 19 municípios estudados, nos anos de 2000 a 2002, podem ser verificados na Tabela 1 . No período analisado, quatro ETAS extrapolaram, pelo menos uma vez, os níveis máximos de trialometanos permitidos pela legislação sanitária do país. No ano de 1998, as ETAs Rio Grande e Casa Grande apresentaram níveis de trialometa-

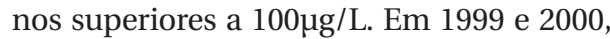
o mesmo comportamento foi registrado na ETA Alto Tietê, e, em 2000 e 2001, no Alto Cotia. Observa-se que a ETA Guaraú apresentou

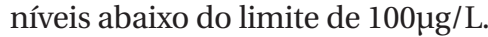

A prevalência de prematuridade nos municípios estudados passou de 4,5\% em 1998 para $7,2 \%$ em 1999, mantendo-se no patamar de 6 a $7 \%$ no restante do período. $\mathrm{O}$ baixo peso ao nascer apresentou comportamento em declínio, passando de 6,3\% para $5,0 \%$, semelhantemente ao observado na prevalência dos defeitos do tubo neural, que passou de $0,4 \%$ em 2000 para $0,04 \%$ em 2002. Já a prevalência para defeito no sistema nervoso central e malformação congênita sofreu pequena variação, tendo aumentado entre 2000 e 2001 e depois diminuído entre 2001 e 2002, voltando aos patamares obtidos em 2000 (Tabela 2).

São Caetano do Sul (9,7\%) e Itapecerica da Serra $(8 \%)$ se destacam por apresentarem os maiores percentuais de prematuridade, Carapicuíba $(6,9 \%)$ pelo maior percentual de baixo peso ao nascer, enquanto Diadema $(0,8 \%)$ e São Caetano do Sul $(0,8 \%)$ apresentam os mais altos percentuais de malformações congênitas (Tabela 3).

Tabela 1 - Valores máximos de trialometanos ( $\mu \mathrm{g} / \mathrm{L}$ ) observados na ETAs da Região Metropolitana de São Paulo de 2000 a 2002.

Table 1 - Maximum trihalomethane values $(\mu \mathrm{g} / \mathrm{L})$ registered at water treatment stations of the metropolitan area of Sao Paulo from 2000 to 2002.

\begin{tabular}{|c|c|c|c|c|}
\hline \multirow[t]{3}{*}{ ETA } & \multirow[t]{3}{*}{ Municípios } & \multicolumn{3}{|c|}{ Trialometanos $\mu \mathrm{g} / \mathrm{L}$} \\
\hline & & \multicolumn{3}{|c|}{ ANO } \\
\hline & & 2000 & 2001 & 2002 \\
\hline \multirow[t]{5}{*}{ Guaraú } & Carapicuíba & 68,5 & 66,0 & 42,0 \\
\hline & Francisco Morato & 41,0 & 34,0 & 47,0 \\
\hline & Francisco Rocha & 40,0 & 42,0 & 50,0 \\
\hline & São Caetano do Sul & $\ldots$ & $\ldots$ & $\ldots$ \\
\hline & Osasco & 35,0 & 26,5 & 31,8 \\
\hline \multirow[t]{3}{*}{ Alto Cotia } & Embu Guaçu & 57,0 & 65,0 & 58,0 \\
\hline & Itapecerica da Serra & 119,0 & 84,0 & 109,0 \\
\hline & Vargem Grande Paulista & 83,2 & 39,0 & $<5,0$ \\
\hline Alto da Boa Vista & Taboão da Serra & 86,0 & 85,0 & 78,0 \\
\hline \multirow[t]{4}{*}{ Alto Tietê } & Diadema & $\ldots$ & $\ldots$ & $\ldots$ \\
\hline & Ferraz Vasconcelos & 92,7 & 88 & 146,0 \\
\hline & Itaquaquecetuba & 80,9 & 152,0 & 96,0 \\
\hline & Poá & 115,0 & 92,0 & 112,0 \\
\hline Rio Grande & São Bernardo do Campo & $\ldots$ & $\ldots$ & $\ldots$ \\
\hline \multirow[t]{2}{*}{ Baixo Cotia } & Itapevi & 79,2 & 79,0 & $\ldots$ \\
\hline & Jandira & $\ldots$ & $\ldots$ & $\ldots$ \\
\hline Casa Grande & Ribeirão Pires & 70,8 & 68,5 & 91,5 \\
\hline
\end{tabular}

Fonte:SABESP, 2003/Source: SABESP, 2003 
Tabela 2 - Prevalência da prematuridade e baixo peso no período de 1998 e 2002 e da malformação congênita, defeito no tubo neural e defeito no SNC no período de 2000 a 2002 nos municípios estudados.

Table 2 - Prevalence of preterm delivery and low birth weight from 1998 to 2002 and of congenital anomalies, neural tube and nervous system defects from 2000 to 2002 in the municipalities studied.

\begin{tabular}{|c|c|c|c|c|c|c|c|c|c|}
\hline \multirow{3}{*}{ Desfecho } & \multirow{3}{*}{$\begin{array}{l}\text { Categorias de } \\
\text { TRIALOMETANOS } \\
\text { (g/L) }\end{array}$} & \multicolumn{4}{|c|}{ Não Ajustado } & \multicolumn{4}{|c|}{ Ajustado } \\
\hline & & \multirow{2}{*}{$\begin{array}{l}\text { Odds } \\
\text { Ratio }\end{array}$} & \multicolumn{2}{|c|}{ IC (95\%) } & \multirow{2}{*}{$P^{*}$} & \multirow{2}{*}{$\begin{array}{l}\text { Odds } \\
\text { Ratio }\end{array}$} & \multicolumn{2}{|c|}{ IC (95\%) } & \multirow{2}{*}{$\mathrm{P}^{*}$} \\
\hline & & & Inferior & Superior & & & Inferior & Superior & \\
\hline \multirow{5}{*}{ Prematuridade ${ }^{a}$} & $<40$ & 1,00 & & & \multirow{5}{*}{0,056} & 1,00 & & & \multirow{5}{*}{$<0,001$} \\
\hline & $>=40 \mathrm{e}<60$ & 1,11 & 1,07 & 1,16 & & 1,08 & 1,03 & 1,13 & \\
\hline & $>=60$ e $<80$ & 1,09 & 1,05 & 1,13 & & 1,10 & 1,06 & 1,15 & \\
\hline & $>=80 \mathrm{e}<100$ & 0,94 & 0,89 & 1,00 & & 0,99 & 0,93 & 1,06 & \\
\hline & $>=100$ & 1,07 & 1,01 & 1,14 & & 1,11 & 1,04 & 1,19 & \\
\hline \multirow{5}{*}{$\begin{array}{l}\text { Malformação } \\
\text { Congênita }^{\mathrm{b}}\end{array}$} & $<40$ & 1,00 & 1,00 & & \multirow{5}{*}{$<0,001$} & & & & \multirow{5}{*}{$<0,001$} \\
\hline & $>=40 \mathrm{e}<60$ & 1,49 & 1,24 & 1,79 & & 1,49 & 1,24 & 1,79 & \\
\hline & $>=60$ e $<80$ & 1,61 & 1,36 & 1,92 & & 1,57 & 1,32 & 1,87 & \\
\hline & $>=80$ e $<100$ & 1,92 & 1,51 & 2,44 & & 1,91 & 1,50 & 2,44 & \\
\hline & $>=100$ & 1,45 & 1,06 & 1,97 & & 1,47 & 1,08 & 2,00 & \\
\hline \multirow{5}{*}{$\begin{array}{l}\text { Defeito no Sistema } \\
\text { Nervoso Centralc }\end{array}$} & $<40$ & 1,00 & & & & 1,00 & & & \multirow{5}{*}{0,538} \\
\hline & $>=40$ e $<60$ & 1,26 & 0,81 & 1,95 & \multirow{4}{*}{0,336} & 1,06 & 0,66 & 1,70 & \\
\hline & $>=60$ e $<80$ & 1,17 & 0,76 & 1,80 & & 1,05 & 0,66 & 1,65 & \\
\hline & $>=80$ e $<100$ & 1,13 & 0,57 & 2,23 & & 1,01 & 0,49 & 2,06 & \\
\hline & $>=100$ & 1,44 & 0,71 & 2,93 & & 1,40 & 0,68 & 2,88 & \\
\hline \multirow{5}{*}{$\begin{array}{l}\text { Defeito no Tubo } \\
\text { Neural }^{d}\end{array}$} & $<40$ & 1,00 & & & \multirow{5}{*}{0,950} & 1,00 & & & \multirow{5}{*}{0,944} \\
\hline & $>=40 \mathrm{e}<60$ & 1,36 & 0,80 & 2,29 & & 1,37 & 0,81 & 2,33 & \\
\hline & $>=60 \mathrm{e}<80$ & 0,96 & 0,55 & 1,68 & & 0,90 & 0,51 & 1,59 & \\
\hline & $>=80$ e $<100$ & 1,00 & 0,42 & 2,40 & & 1,04 & 0,43 & 2,48 & \\
\hline & $>=100$ & 1,19 & 0,46 & 3,04 & & 1,29 & 0,50 & 3,31 & \\
\hline \multirow{5}{*}{ Baixo Peso } & $<40$ & 1,00 & & & \multirow{5}{*}{0,045} & 1,00 & & & \multirow{5}{*}{0,012} \\
\hline & $>=40$ e $<60$ & 0,92 & 0,88 & 0,96 & & 0,92 & 0,87 & 0,97 & \\
\hline & $>=60$ e $<80$ & 0,93 & 0,90 & 0,97 & & 0,94 & 0,89 & 0,98 & \\
\hline & $>=80 \mathrm{e}<100$ & 0,96 & 0,90 & 1,02 & & 0,92 & 0,86 & 0,99 & \\
\hline & $>=100$ & 0,98 & 0,91 & 1,05 & & 0,95 & 0,88 & 1,03 & \\
\hline
\end{tabular}

Fonte: Dados de pesquisa/Source: Study data

As malformações congênitas mostraram associação estatisticamente significativa com as covariáveis sexo, tipo de gravidez, tipo de parto, faixa etária da mãe e exposição ao trialometano (em concentrações superiores a $100 \mu \mathrm{g} / \mathrm{L}$ ). Tipo de parto e tipo de gravidez apresentaram associação com defeitos no Sistema Nervoso Central e defeitos no tubo neural.

Observou-se que as covariáveis, sexo do $\mathrm{RN}$, tipo de parto, tipo de gravidez, escolari- dade e idade da mãe, realização de pré-natal, bem como a presença de trialometanos na água estiveram associadas estatisticamente com prematuridade. Observa-se ainda associação estatisticamente significativa entre todas as covariáveis estudadas e o baixo peso ao nascer (Tabela 2).

Nos modelos multivariados, em cada trimestre gestacional, os desfechos foram ajustados pelas características maternas, do recém-nascido, da gestação e do parto, 
Tabela 3 - Frequência da prematuridade, baixo peso, malformações congênitas, defeito do sistema nervoso central e defeito no tubo neural nos municípios estudados.

Table 3 - Frequency of prematurity, low birth weight, congenital anomalies, neural tube and nervous system defects in the municipalities studied.

\begin{tabular}{|c|c|c|c|c|c|c|c|c|c|c|c|c|c|c|c|}
\hline \multirow[t]{2}{*}{ Município } & \multicolumn{3}{|c|}{ Prematuridade } & \multicolumn{3}{|c|}{ Baixo peso } & \multicolumn{3}{|c|}{$\begin{array}{l}\text { Malformações } \\
\text { Congênitas }\end{array}$} & \multicolumn{3}{|c|}{ Defeito do SNC } & \multicolumn{3}{|c|}{ Defeito do tubo neural } \\
\hline & Sim & (\%) & Total & Sim & (\%) & Total & Sim & (\%) & Total & Sim & $(\%)$ & Total & Sim & (\%) & Total \\
\hline Carapicuíba & 2327 & 6,8 & 34269 & 2181 & 6,9 & 31787 & 78 & 0,4 & 19338 & 13 & 0,1 & 19334 & 8 & 0,0 & 19334 \\
\hline Diadema & 3029 & 7,4 & 41192 & 1954 & 5,1 & 37979 & 178 & 0,8 & 22828 & 21 & 0,1 & 22827 & 16 & 0,1 & 22827 \\
\hline Embu-Guaçu & 395 & 7,1 & 5602 & 302 & 5,8 & 5171 & 13 & 0,4 & 3147 & 2 & 0,1 & 3146 & 1 & 0,0 & 3146 \\
\hline $\begin{array}{l}\text { Ferraz de } \\
\text { Vasconcelos }\end{array}$ & 743 & 4,6 & 16284 & 963 & 6,2 & 15515 & 16 & 0,2 & 9001 & 2 & 0,0 & 9000 & 2 & 0,0 & 9000 \\
\hline Francisco Morato & 680 & 4,4 & 15503 & 971 & 6,6 & 14788 & 17 & 0,2 & 8418 & 5 & 0,1 & 8418 & 4 & 0,0 & 8418 \\
\hline Franco da Rocha & 719 & 6,3 & 11457 & 709 & 6,6 & 10686 & 34 & 0,5 & 6308 & 7 & 0,1 & 6307 & 5 & 0,1 & 6307 \\
\hline Itapecerica da Serra & 1432 & 8,0 & 17902 & 796 & 4,8 & 16422 & 27 & 0,3 & 10641 & 5 & 0,0 & 10640 & 0 & 0,0 & 10640 \\
\hline Itapevi & 1305 & 7,0 & 18575 & 1080 & 6,3 & 17202 & 36 & 0,3 & 10500 & 8 & 0,1 & 10499 & 8 & 0,1 & 10499 \\
\hline Itaquaquecetuba & 1906 & 6,5 & 29522 & 1688 & 6,1 & 27573 & 105 & 0,6 & 17273 & 18 & 0,1 & 17270 & 10 & 0,1 & 17270 \\
\hline Jandira & 489 & 4,7 & 10498 & 623 & 6,3 & 9957 & 17 & 0,3 & 5741 & 1 & 0,0 & 5739 & 0 & 0,0 & 5739 \\
\hline Osasco & 3472 & 5,3 & 65114 & 3545 & 5,8 & 61395 & 80 & 0,2 & 35726 & 16 & 0,0 & 35725 & 12 & 0,0 & 35725 \\
\hline Poá & 422 & 4,4 & 9634 & 569 & 6,2 & 9194 & 16 & 0,3 & 5365 & 3 & 0,1 & 5365 & 3 & 0,1 & 5365 \\
\hline Ribeirão Pires & 462 & 5,0 & 9220 & 487 & 5,6 & 8737 & 12 & 0,2 & 5210 & 5 & 0,1 & 5209 & 1 & 0,0 & 5209 \\
\hline $\begin{array}{l}\text { São Bernardo do } \\
\text { Campo }\end{array}$ & 4151 & 6,5 & 63459 & 2848 & 4,8 & 59124 & 241 & 0,7 & 35245 & 28 & 0,1 & 35245 & 18 & 0,1 & 35245 \\
\hline São Caetano do Sul & 826 & 9,7 & 8536 & 384 & 5,0 & 7650 & 37 & 0,8 & 4853 & 6 & 0,1 & 4853 & 3 & 0,1 & 4853 \\
\hline Taboão da Serra & 1517 & 6,0 & 25118 & 1192 & 5,1 & 23544 & 84 & 0,6 & 13897 & 15 & 0,1 & 13894 & 9 & 0,1 & 13894 \\
\hline $\begin{array}{l}\text { Vargem Grande } \\
\text { Paulista }\end{array}$ & 269 & 7,8 & 3443 & 154 & 4,9 & 3168 & 5 & 0,2 & 2052 & 1 & 0,0 & 2052 & 1 & 0,0 & 2052 \\
\hline Total & 24144 & 6,3 & 385328 & 20446 & 5,7 & 359892 & 996 & 0,5 & 215543 & 156 & 0,1 & 215523 & 101 & 0,0 & 215523 \\
\hline
\end{tabular}

Fonte: Dados de pesquisa/Source: Study data

entendendo os trialometanos como medidas categóricas.

No primeiro trimestre de gestação, os desfechos: prematuridade, malformação congênita e baixo peso ao nascer apresentaram associação independente para o consumo de água com trialometanos (os cálculos da associação entre trialometanos presentes na água e as características dos recém-nascidos foram feitos com base no modelo de regressão múltipla não condicional). Quanto maior sua concentração na água de consumo, maior a chance de nascimento de prematuro e ocorrência de malformação congênita, no entanto, a presença de trialometanos significou um fator de proteção para o baixo peso ao nascer. Observou-se que o aumento de concentração destes com- postos diminui a possibilidade do recémnascido apresentar o desfecho analisado (Tabela 4).

Malformação congênita e baixo peso ao nascer apresentaram associação independente com presença de trialometanos na água consumida durante o segundo trimestre gestacional. O aumento da concentração desses compostos aumenta a chance de os recém-nascidos apresentarem malformação congênita e aumenta o efeito de proteção para baixo peso ao nascer conforme observado na Tabela 5 .

A presença de trialometanos na água consumida no último trimestre de gestação mostrou associação semelhante à identificada no segundo trimestre. O aumento da concentração destes compostos (Tabela 6) aumenta a chance da presença de mal- 
Tabela 4 - Associação entre trialometanos presentes na água consumida no primeiro trimestre de gestação e características dos recém-nascidos.

Table 4 - The association between trihalomethanes present in the water consumed during the first trimester of pregnancy and characteristics of newborns.

\begin{tabular}{|c|c|c|c|c|c|c|c|c|c|}
\hline \multirow{3}{*}{ Desfecho } & \multirow{3}{*}{$\begin{array}{l}\text { Categorias de } \\
\text { THM }(\mu \mathrm{g} / \mathrm{L})\end{array}$} & \multicolumn{4}{|c|}{ Não Ajustado } & \multicolumn{4}{|c|}{ Ajustado } \\
\hline & & \multirow{2}{*}{$\begin{array}{l}\text { Odds } \\
\text { Ratio }\end{array}$} & \multicolumn{2}{|c|}{ IC (95\%) } & \multirow{2}{*}{$\mathrm{P}^{*}$} & \multirow{2}{*}{$\begin{array}{l}\text { Odds } \\
\text { Ratio }\end{array}$} & \multicolumn{2}{|c|}{ IC $(95 \%)$} & \multirow{2}{*}{$\mathrm{p}^{*}$} \\
\hline & & & Inferior & Superior & & & Inferior & Superior & \\
\hline \multirow{5}{*}{ Mal Formação Congênitaa } & $<40$ & 1,00 & & & \multirow{5}{*}{$<0,001$} & 1,00 & & & \multirow{5}{*}{$<0,001$} \\
\hline & $>=40$ e $<60$ & 1,38 & 1,09 & 1,73 & & 1,37 & 1,08 & 1,72 & \\
\hline & $>=60$ e $<80$ & 1,79 & 1,49 & 2,15 & & 1,74 & 1,45 & 2,09 & \\
\hline & $>=80$ e $<100$ & 1,57 & 1,18 & 2,10 & & 1,54 & 1,15 & 2,06 & \\
\hline & $>=100$ & 1,29 & 0,89 & 1,86 & & 1,27 & 0,87 & 1,85 & \\
\hline \multirow{5}{*}{$\begin{array}{l}\text { Defeito no Sistema Nervoso } \\
\text { Central }^{\mathrm{b}}\end{array}$} & $<40$ & 1,00 & & & \multirow{5}{*}{0,852} & 1,00 & & & \multirow{5}{*}{0,612} \\
\hline & $>=40$ e $<60$ & 0,83 & 0,44 & 1,55 & & 0,76 & 0,39 & 1,47 & \\
\hline & $>=60$ e $<80$ & 1,36 & 0,88 & 2,12 & & 1,24 & 0,77 & 2,00 & \\
\hline & $>=80$ e $<100$ & 0,55 & 0,20 & 1,53 & & 0,44 & 0,13 & 1,42 & \\
\hline & $>=100$ & 0,61 & 0,19 & 1,98 & & 0,62 & 0,19 & 2,02 & \\
\hline \multirow{5}{*}{ Defeito no Tubo Neural ${ }^{c}$} & $<40$ & 1,00 & & & \multirow{5}{*}{0,516} & 1,00 & & & \multirow{5}{*}{0,177} \\
\hline & $>=40$ e $<60$ & 0,62 & 0,27 & 1,42 & & 0,61 & 0,26 & 1,42 & \\
\hline & $>=60 \mathrm{e}<80$ & 1,24 & 0,72 & 2,11 & & 1,09 & 0,61 & 1,94 & \\
\hline & $>=80$ e $<100$ & 0,57 & 0,17 & 1,88 & & 0,41 & 0,10 & 1,73 & \\
\hline & $>=100$ & 0,28 & 0,04 & 2,09 & & - & & & \\
\hline \multirow{5}{*}{ Baixo Peso ${ }^{d}$} & $<40$ & 1,00 & & & \multirow{5}{*}{$<0,001$} & 1,00 & & & \multirow{5}{*}{0,003} \\
\hline & $>=40$ e $<60$ & 0,91 & 0,87 & 0,95 & & 0,89 & 0,84 & 0,94 & \\
\hline & $>=60$ e $<80$ & 0,91 & 0,87 & 0,94 & & 0,92 & 0,88 & 0,96 & \\
\hline & $>=80$ e $<100$ & 0,90 & 0,85 & 0,96 & & 0,90 & 0,84 & 0,97 & \\
\hline & $>=100$ & 0,96 & 0,90 & 1,03 & & 0,97 & 0,90 & 1,05 & \\
\hline
\end{tabular}

Fonte: Dados de pesquisa/ Source: Study data.

Nota: *teste de tendência/Note:*trend test.

a ajustado para as variáveis: tipo de gravidez, tipo de parto, sexo do RN e faixa etária da mãe.

${ }^{\text {b }}$ ajustado para as variáveis: tipo parto, tipo de gravidez, faixa etária da mãe e o sexo do RN.

cajustado para as variáveis: tipo de parto, tipo de gravidez, escolaridade da mãe e faixa etária da mãe.

dajustado para as variáveis: sexo, tipo de gravidez, tipo de parto, pré-natal e faixa etária da mãe.

${ }^{a}$ adjusted for variables: type of pregnancy, type of delivery, sex of NB, and mother age group.

${ }^{b}$ adjusted for variables: type of delivery, type of pregnancy, mother age group, and sex of $N B$.

' adjusted for variables: type of delivery, type of pregnancy, mother's schooling, and mother age group.

${ }^{d}$ adjusted for variables: sex, type of pregnancy, type of delivery, pre-natal, and mother age group.

formação congênita nos recém-nascidos e aumenta o efeito de proteção para nascimentos de baixo peso.

\section{Discussão}

Um dos problemas encontrados por pesquisas desta natureza é a coexistência de muitos fatores de confusão, e limitações de causas diversas, uma vez que se trabalhou com uma região muito extensa e densamente populosa e uma vez que os problemas relacionados à gravidez podem estar relacionados a diferentes fatores.

A pesquisa comportou vieses de informação e de seleção. $O$ viés de informação relaciona-se:

- à falta de dados precisos sobre a exposição, decorrente, em parte, da dificuldade na obtenção dos dados relativos aos níveis de trialometanos na água de abastecimento, os quais apresentam 
Tabela 5 - Associação entre trialometanos presentes na água consumida no segundo trimestre de gestação e características dos recém-nascidos.

Table 5 - The association between trihalomethanes present in the water consumed during the second trimester of pregnancy and characteristics of newborns.

\begin{tabular}{|c|c|c|c|c|c|c|c|c|c|}
\hline \multirow{3}{*}{ Desfecho } & \multirow{3}{*}{$\begin{array}{l}\text { Categorias de } \\
\mathrm{THM}(\mu \mathrm{g} / \mathrm{L})\end{array}$} & \multicolumn{4}{|c|}{ Não Ajustado } & \multicolumn{4}{|c|}{ Ajustado } \\
\hline & & \multirow{2}{*}{$\begin{array}{l}\text { Odds } \\
\text { Ratio }\end{array}$} & \multicolumn{2}{|c|}{ IC (95\%) } & \multirow[t]{2}{*}{$\mathbf{P}^{*}$} & \multirow{2}{*}{$\begin{array}{l}\text { Odds } \\
\text { Ratio }\end{array}$} & \multicolumn{2}{|c|}{ IC (95\%) } & \multirow{2}{*}{$\mathbf{p}^{*}$} \\
\hline & & & Inferior & Superior & & & Inferior & $\overline{\text { Superior }}$ & \\
\hline \multirow{5}{*}{ Mal Formação Congênitaa } & $<40$ & 1,00 & & & \multirow{5}{*}{$<0,001$} & 1,00 & & & \multirow{5}{*}{$<0,001$} \\
\hline & $>=40$ e $<60$ & 1,23 & 0,97 & 1,56 & & 1,21 & 0,95 & 1,54 & \\
\hline & $>=60$ e $<80$ & 1,52 & 1,27 & 1,82 & & 1,49 & 1,24 & 1,79 & \\
\hline & $>=80 \mathrm{e}<100$ & 1,82 & 1,42 & 2,33 & & 1,77 & 1,38 & 2,27 & \\
\hline & $>=100$ & 1,36 & 0,97 & 1,91 & & 1,36 & 0,96 & 1,92 & \\
\hline \multirow{5}{*}{$\begin{array}{l}\text { Defeito no Sistema Nervoso } \\
\text { Central }^{b}\end{array}$} & $<40$ & 1,00 & & & & 1,00 & & & \multirow{5}{*}{0,517} \\
\hline & $>=40$ e $<60$ & 1,06 & 0,58 & 1,94 & \multirow{4}{*}{0,321} & 1,13 & 0,61 & 2,10 & \\
\hline & $>=60 \mathrm{e}<80$ & 1,18 & 0,74 & 1,89 & & 1,11 & 0,67 & 1,84 & \\
\hline & $>=80 \mathrm{e}<100$ & 1,19 & 0,59 & 2,40 & & 1,16 & 0,55 & 2,44 & \\
\hline & $>=100$ & 1,43 & 0,64 & 3,20 & & 1,33 & 0,55 & 3,20 & \\
\hline \multirow{5}{*}{ Defeito no Tubo Neuralc } & $<40$ & 1,00 & & & \multirow{5}{*}{0,729} & 1,00 & & & \multirow{5}{*}{0,890} \\
\hline & $>=40$ e $<60$ & 1,01 & 0,49 & 2,09 & & 1,10 & 0,51 & 2,36 & \\
\hline & $>=60$ e $<80$ & 1,07 & 0,60 & 1,90 & & 1,04 & 0,56 & 1,94 & \\
\hline & $>=80 \mathrm{e}<100$ & 1,36 & 0,62 & 3,00 & & 1,12 & 0,45 & 2,80 & \\
\hline & $>=100$ & 0,87 & 0,26 & 2,88 & & 0,68 & 0,16 & 2,90 & \\
\hline \multirow{5}{*}{ Baixo Peso ${ }^{d}$} & $<40$ & 1,00 & & & \multirow{5}{*}{$<0,001$} & 1,00 & & & \multirow{5}{*}{$<0,001$} \\
\hline & $>=40$ e $<60$ & 0,91 & 0,87 & 0,95 & & 0,89 & 0,85 & 0,93 & \\
\hline & $>=60$ e $<80$ & 0,87 & 0,84 & 0,90 & & 0,87 & 0,83 & 0,91 & \\
\hline & $>=80$ e $<100$ & 0,98 & 0,92 & 1,03 & & 0,96 & 0,90 & 1,03 & \\
\hline & $>=100$ & 0,86 & 0,80 & 0,92 & & 0,85 & 0,78 & 0,92 & \\
\hline
\end{tabular}

Fonte: Dados de pesquisa Source: Study data.

Nota: *teste de tendência/Note: *tendency test.

a ajustado para as variáveis: tipo de gravidez, tipo de parto, sexo do RN e faixa etária da mãe.

b ajustado para as variáveis: tipo parto, tipo de gravidez, faixa etária da mãe e o sexo do RN.

cajustado para as variáveis: tipo de parto, tipo de gravidez, escolaridade da mãe e faixa etária da mãe.

${ }^{d}$ ajustado para as variáveis: sexo, tipo de gravidez, tipo de parto, pré-natal e faixa etária da mãe.

${ }^{a}$ adjusted for variables: type of pregnancy, type of delivery, sex of NB, and mother age group.

${ }^{b}$ adjusted for variables: type of delivery, type of pregnancy, mother age group, and sex of NB.

' adjusted for variables: type of delivery, type of pregnancy, mother's schooling, and mother age group.

${ }^{d}$ adjusted for variables: sex, type of pregnancy, type of delivery, pre-natal, and mother age group.

disponibilidade preservada e não eram sistematizados;

- a exposição dos sujeitos aos trialometanos foi definida de forma ecológica, baseada no nível máximo de trialometanos mensurado na ETA que abastecia a residência; no entanto, essa não corresponde necessariamente à concentração de trialometanos na água utilizada nos domicílios, em virtude de alterações a que a água pode estar sujeita no trajeto entre a ETA e os domicílios;

- à impossibilidade de mensuração exata do grau de exposição da mãe aos trialometanos devido à mobilidade da residência materna e à possibilidade de a mãe ter consumido água em outros lugares além da residência, bem como devido à incerteza sobre a origem e a quantidade de água ingerida pela gestante; 
Tabela 6 - Associação entre trialometanos presentes na água consumida no terceiro trimestre de gestação e característica dos recém nascidos.

Table 6 - The association between trihalomethanes present in the water consumed during the third trimester of pregnancy and the characteristics of newborns.

\begin{tabular}{|c|c|c|c|c|c|c|c|c|c|}
\hline \multirow{3}{*}{ Desfecho } & \multirow{3}{*}{$\begin{array}{l}\text { Categorias de } \\
\text { THM }(\mu \mathrm{g} / \mathrm{L})\end{array}$} & \multicolumn{4}{|c|}{ Não Ajustado } & \multicolumn{4}{|c|}{ Ajustado } \\
\hline & & \multirow{2}{*}{$\begin{array}{l}\text { Odds } \\
\text { Ratio }\end{array}$} & \multicolumn{2}{|c|}{ IC (95\%) } & \multirow[t]{2}{*}{$\mathrm{P}^{*}$} & \multirow{2}{*}{$\begin{array}{l}\text { Odds } \\
\text { Ratio }\end{array}$} & \multicolumn{2}{|c|}{ IC (95\%) } & \multirow[t]{2}{*}{$\mathrm{p}^{*}$} \\
\hline & & & Inferior & Superior & & & Inferior & Superior & \\
\hline \multirow{5}{*}{$\begin{array}{l}\text { Mal Formação } \\
\text { Congênitaa }\end{array}$} & $<40$ & 1,00 & & & \multirow{5}{*}{$<0,001$} & 1,00 & & & \multirow{5}{*}{$<0,001$} \\
\hline & $>=40 \mathrm{e}<60$ & 1,23 & 0,97 & 1,56 & & 1,21 & 0,95 & 1,54 & \\
\hline & $>=60$ e $<80$ & 1,52 & 1,27 & 1,82 & & 1,49 & 1,24 & 1,79 & \\
\hline & $>=80 \mathrm{e}<100$ & 1,82 & 1,42 & 2,33 & & 1,77 & 1,38 & 2,27 & \\
\hline & $>=100$ & 1,36 & 0,97 & 1,91 & & 1,36 & 0,96 & 1,92 & \\
\hline \multirow{5}{*}{$\begin{array}{l}\text { Defeito no Sistema } \\
\text { Nervoso Central }^{b}\end{array}$} & $<40$ & 1,00 & & & & 1,00 & & & \multirow{5}{*}{0,517} \\
\hline & $>=40 \mathrm{e}<60$ & 1,06 & 0,58 & 1,94 & \multirow{4}{*}{0,321} & 1,13 & 0,61 & 2,10 & \\
\hline & $>=60$ e $<80$ & 1,18 & 0,74 & 1,89 & & 1,11 & 0,67 & 1,84 & \\
\hline & $>=80 \mathrm{e}<100$ & 1,19 & 0,59 & 2,40 & & 1,16 & 0,55 & 2,44 & \\
\hline & $>=100$ & 1,43 & 0,64 & 3,20 & & 1,33 & 0,55 & 3,20 & \\
\hline \multirow{5}{*}{ Defeito no Tubo Neuralc } & $<40$ & 1,00 & & & \multirow{5}{*}{0,729} & 1,00 & & & \multirow{5}{*}{0,890} \\
\hline & $>=40$ e $<60$ & 1,01 & 0,49 & 2,09 & & 1,10 & 0,51 & 2,36 & \\
\hline & $>=60$ e $<80$ & 1,07 & 0,60 & 1,90 & & 1,04 & 0,56 & 1,94 & \\
\hline & $>=80 \mathrm{e}<100$ & 1,36 & 0,62 & 3,00 & & 1,12 & 0,45 & 2,80 & \\
\hline & $>=100$ & 0,87 & 0,26 & 2,88 & & 0,68 & 0,16 & 2,90 & \\
\hline \multirow{5}{*}{ Baixo Peso ${ }^{d}$} & $<40$ & 1,00 & & & \multirow{5}{*}{$<0,001$} & 1,00 & & & \multirow{5}{*}{$<0,001$} \\
\hline & $>=40$ e $<60$ & 0,91 & 0,87 & 0,95 & & 0,89 & 0,85 & 0,93 & \\
\hline & $>=60 \mathrm{e}<80$ & 0,87 & 0,84 & 0,90 & & 0,87 & 0,83 & 0,91 & \\
\hline & $>=80 \mathrm{e}<100$ & 0,98 & 0,92 & 1,03 & & 0,96 & 0,90 & 1,03 & \\
\hline & $>=100$ & 0,86 & 0,80 & 0,92 & & 0,85 & 0,78 & 0,92 & \\
\hline
\end{tabular}

Fonte: Dados de pesquisa/Source: Study data.

Nota: *teste de tendência/Note: *tendency test.

a ajustado para as variáveis: tipo de gravidez, tipo de parto, sexo do RN e faixa etária da mãe.

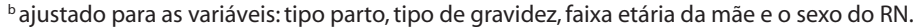

cajustado para as variáveis: tipo de parto, tipo de gravidez, escolaridade da mãe e faixa etária da mãe.

dajustado para as variáveis: sexo, tipo de gravidez, tipo de parto, pré-natal e faixa etária da mãe.

${ }^{a}$ adjusted for variables: type of pregnancy, type of delivery, sex of NB, and mother age group.

${ }^{b}$ adjusted for variables: type of delivery, type of pregnancy, mother age group, and sex of $N B$.

' adjusted for variables: type of delivery, type of pregnancy, mother's schooling, and mother age group.

${ }^{d}$ adjusted for variables: sex, type of pregnancy, type of delivery, pre-natal, and mother age group.

- à não verificação do reservatório domiciliar, caixa d'água, o qual pode apresentar diferentes condições de conservação, que propiciem ou não acúmulo de matéria orgânica (conteúdo fecal de roedores, roedores mortos, lodo etc.).

Com relação a malformações congênitas cujos valores encontrados situaram-se bem abaixo dos valores citados pelo Ministério da Saúde para o Brasil, entre 2 e 3\%, é importante anotar a deficiência na identificação das malformações congênitas e ou anomalias cromossômicas no momento do nascimento, favorecendo o sub-registro. Outrossim, apenas em 2000, dados a respeito de malformações congênitas foram incluídos na DN.

$\mathrm{O}$ fato de a prevalência média encontrada para prematuridade (de $6,3 \%$ ) ter se 
encontrado abaixo do valor apontado pelo levantamento nacional brasileiro para o estado de São Paulo, situado em 8,9\% para o ano de $1998^{25}$ e também abaixo do valor encontrado por Kilsztajn, Back, Carmo e Sugahara ${ }^{26}$ para o Estado de São Paulo, situado em $7,3 \%$ para o ano de 2000 , talvez se deva a problemas relacionados aos dados coletados no SINASC, o que pode ser denotado pela diferença entre o valor de prevalência de prematuridade encontrado para o ano de 1998 e os valores obtidos para os demais anos.

A prevalência média de baixo peso situou-se em 5,6\%, variando entre 6,3\% e 5,0\% de 1998 a 2002. As prevalências de baixo peso estiveram todas abaixo de $15 \%$, índice estimado pelo Ministério da Saúde ${ }^{25}$ para o Brasil no ano 2000, e abaixo de 8,8\%, índice estimado por Kilsztajn, Back, Carmo e Sugahara ${ }^{26}$ para o Estado de São Paulo.

Para os Defeitos no Tubo Neural e Sistema Nervoso Central, os índices encontrados estiveram abaixo dos esperados, provavelmente por uma questão de diagnóstico. Analisando o triênio 2000-2002, observa-se uma redução significativa dos índices aqui obtidos, o que se diferencia da literatura pesquisada.

A associação evidenciada nesta pesquisa entre os trialometanos, a prematuridade e malformações congênitas, durante o primeiro trimestre da gestação, encontra-se em discordância com os dados encontrados na literatura que, à exceção de Kramer, Lynch, Isacson e Hanson ${ }^{11}$, apontaram para uma não associação dos trialometanos com prematuridade.

O fato de os trialometanos terem se apresentado como fator de proteção para o desfecho baixo peso, no primeiro trimestre da gravidez, contraria os resultados de diversos outros estudos que apontam para uma relação de risco entre trialometanos e baixo peso ${ }^{11,12,14}$. No entanto, também em estudos internacionais relativos ao desfecho baixo peso percebem-se discordâncias que parecem estar relacionadas à metodologia de coleta de dados pela qual se optou em cada um dos casos.
No que se refere a viés de seleção, entendemos que, uma vez que todos os nascidos vivos foram incluídos na pesquisa, esse não deve ter constituído um problema na definição dos resultados de pesquisa. É relevante também mencionar possíveis variáveis de confusão como, estado clínico-nutricional das mães, uso de drogas, psicofármacos ou álcool, tabagismo, exposição da mãe a toxinas ambientais, história genética dos pais, abortos prévios, as quais não foram controladas.

Observou-se uma sazonalidade nos níveis de trialometanos presentes na água das ETAs durante o período estudado, sendo os níveis mais elevados registrados durante os meses de outubro a março, período de precipitação mais elevada em São Paulo, o que potencializa o arraste de substâncias húmicas e fúlvicas para os rios e, consequentemente, para os reservatórios ${ }^{5}$.

A partir da análise dos dados coletados durante a pesquisa percebeu-se uma inobservância relativa ao nível máximo de trialometanos fixado pelo Ministério da Saúde para o Brasil, encontrando-se concentrações elevadas destes, ou seja, concentrações $\geq 100 \mu \mathrm{g} / \mathrm{L}$ nas ETAs Alto Cotia nos anos de 2000 e 2001, Alto Tietê nos anos de 1999 e 2000, Rio Grande no ano de 1998 e na ETA Casa Grande no ano de 1998. Esses níveis elevados de trialometanos são similares aos encontrados por Tominaga e Midio $^{1}$ em São Paulo.

Apesar das limitações do trabalho, da dificuldade no controle das variáveis de confusão e dos riscos reduzidos encontrados, os resultados aqui obtidos apontaram para uma associação e um possível risco de malformação e prematuridade relacionado aos trialometanos, o que aponta para necessidade de novas pesquisas a este respeito, dadas as implicações diretas e indiretas de tal fato sobre a Saúde Pública.

A revisão dos resultados de pesquisa e da literatura consultada sugere uma associação entre desfechos adversos na gravidez e a presença de subprodutos resultantes da adição de substâncias químicas à água, visando ao seu tratamento; todavia, não se 
pode deixar de reconhecer o enorme benefício que o uso dessas substâncias implica em termos de saúde pública.

Os países que adotaram a cloração da água como medida de prevenção às doenças infecciosas observaram uma redução das taxas de incidência destas doenças a níveis muito baixos quando comparadas aos países que não a utilizam. Entretanto, os resultados aqui obtidos indicam a necessidade de uma melhor monitoração dos níveis de trialometanos presentes na água de abastecimento público, bem como a urgência de estudos subsequentes que venham a contribuir de forma efetiva para uma reanálise do nível máximo dos trialometanos na água, o qual corresponde a um valor presentemente muito acima do estipulado por outros países onde a pesquisa na área se encontra mais avançada.

\section{Referências}

1. Tominaga MY, Midio AF. Exposição humana a trialometanos presentes em água tratada. Rev Saúde Pública 1999; 33 (4): 413-21.

2. Brasil. FUNASA. Portaria n. 1469/ 2001: Controle e Vigilância da Qualidade da Água para consumo Humano e seu Padrão de Potabilidade; 2001.

3. Riedel G. Controle Sanitário dos Alimentos. 2a ed. São Paulo: Atheneu; 1992.

4. Fernicola NAGG, Azevedo FA. Water levels of trihalomethanes (THM) in four different supply systems from São Paulo, Brazil. J Environ Health 1984; 46: 187-8.

5. OPAS. Programa Especial de Salud Maternoinfantily Población/Programa Ampliado de Inmunización. Boletín Informativo PAI No 6. Washington DC; 1994.

6. Wrensch M, Swan SH, Lipscomb J, Epstein DM, Neutra RR, Fenster L. Spontaneous Abortions and birth defects related to tap and bottled water use. Epidemiology 1992; 3 (2): 98-103.

7. Magnus P et al. Water chlorination and defects. Epidemiology 1999; 10: 513-7.

8. Infant-Rivard C, Olson E, Jacques L, Ayotte P. Drinking water contaminants and childhood leukemia. Epidemiology 2001; 12 (1): 4-6.

9. Toledano MB et al. Relation of trihalomethane concentrations in public water supplies to stillbirth and birth weight in three water regions in England. Environ Health Perspect 2005; 113(2): 225-32.

10. Hawmg BF, Jaakkola JJ. Water chlorination and birth defects: a systematic review and meta-analysis. Environ Health 2003; 58 (2): 83-91.

11. Kramer MD, Lynch CF, Isacson P, Hanson JW. The association of waterborn chloroform with intrauterine growth retardation. Epidemiology 1992; 3: 407-13.

12. Bove FJ, Fulcomer MC, Klotz JB, Esmart J, Dufficy EM, Savrin JE. Public drinking water contamination and birth outcomes. Am J Epidemilogical 1995; 141: 850-62.
13. Waller K, Swan SH, Delorenze G, Hopkins B. Trihalomethanes in drinking water and spontaneous abortion. Epidemiology 1998; 9: 134-40.

14. Gallagher MD, Nuckols JR, Stallones L, Savitz DA, Exposure to trihalomethanes and adverse pregnancy outcomes. Epidemiology 1998; 9: 484-9.

15. Dodds L, King W, Woolcott C, Pole J. Trihalomethanes in public water supplies and adverse birth outcomes. Epidemiology 1999; 10: 233-7.

16. Klotz JB, Pyrch LA. Neural tube defects and drinking water disinfection by-products. Epidemiology 1999; 10: 383-90.

17. Wright JM, Schwartz J, Dockery DW. Effect of trihalomethane exposure on fetal development. Occup Environ Med 2003; 60: 173-80.

18. Dodds L, King W, Allen AC, Armson BA, Fell DB, Nimrod C. Trihalomethanes in public water supplies and risk of stillbirth. Epidemiology 2004; 15 (2): 179-86.

19. Aggazzotti G, Righi E, Fantuzzi g, Biasotti B, Ravera G, Kanitz S et al. Chlorination by-products (CBPs) in drinking water and adverse pregnancy outcomes in Italy. JWater Health 2004; 2 (4): 233-247.

20. Monteiro CA, Benicio MH, Ortiz LP. Secular trends in births weight in the city of São Paulo, Brazil (1976-1998). Rev Saúde Pública 2000; 34 (S6): 26-40.

21. Pereira MG. Epidemiologia teoria e prática. Rio de Janeiro: Guanabara Koogan; 1995.

22. Hay WW, Hayward AR, Levin MJ, Sondheimer JM. Current pediatric diagnosis and treatment. $14^{\text {th }}$ ed. USA: Appleton and Lange; 1999.

23. Selevan GS, Kimmel CA, Mendola P. Identifying critical windows of exposure for children's health. Environ Health Perspect 2000; 108 (S3): 451-55.

24. Hosmer WD, Lemeshow S. Applied Logistic Regression. 2nd ed. New York: John Willey \& Sons; 2000. 
25. BRASIL. Ministério da Saúde. Secretaria de Políticas de Saúde. Gestação de alto risco. Manual Técnico. $3^{\mathrm{a}}$ ed. Brasília: Ministério da Saúde; 2000.

26. Kilsztajn A, Back AC, Carmo MSN do, Sugahara GTL. Assistência pré-natal, baixo-peso e prematuridade no estado de São Paulo. Rev Saúde Pública 2003, 37(3): 303-310.
27. Environmental Working Group (EWG/USPIRG EDUCATION FUND, 2001); 2002. Disponível em http://20009-info@ewg.org. [Acessado em 24 de novembro de 2003].

Recebido em: 26/03/10

Versão final reapresentada em: 04/10/10

Aprovado em: 12/11/10 